\title{
ARTE EFFYMERO. L'APOCALYPSE COMME PRINCIPE DE CRÉATION DANS LA BANDE DESSINÉE DE L'ARTISTE TRANS EFFY BETH
}

\author{
MARIE LORINQUER-HERVÉ \\ Université Bordeaux-Montaigne
}

\begin{abstract}
En 2012, l'artiste trans israélo-argentine Effy Beth inscrit en couverture de son unique bande dessinée, TRANSita rápido - TRANSita lento, la question rhétorique: "2012! Qué no es ahora el fin del mundo?" Selon les interprétations New Age du calendrier maya, l'année 2012 devait en effet être celle de la fin du monde. Placée en ouverture d'un récit de non-fiction qui insiste sur les difficultés quotidiennes imposées aux personnes queer, la question peut toutefois être lue comme davantage qu'un clin d'œil conjoncturel. Dans sa bande dessinée, Effy Beth narre sa transidentité comme une identité apocalyptique. Elle transmue la transgression en principe de création et poursuit l'élaboration d'une poétique et d'une politique de l'éphémère initiée dans ses œuvres précédentes.
\end{abstract}

MOTS CLÉS: Effy Beth, bande dessinée, narrations queer, apocalypse, Argentine.

Arte Effymero. The Apocalypse as a Principle of Creation in the Comic Strip by Transgender Artist Effy Beth

In 2012, the transgender Israeli-Argentinian performance artist Effy Beth wrote in the front page of her only comic strip, TRANSita rápido - TRANSita lento, the rhetorical question: "2012! Qué no es ahora el fin del mundo?" According to the New Age interpretation of the Mayan calendar, 2012 was supposed to mark the end of the world. Associated with a non-fiction narrative that focuses on the everyday challenges queer people suffer from in this society, the question can however be understood as more than a circumstantial reference. In her comic-strip, Effy Beth narrates her transgender identity as an apocalyptic one. She makes transgression into a principle of creation and goes on developing a poetics and a politics of the ephemeral, as she had done in her previous works.

KEY WORDS: Effy Beth, comic strip, queer narratives, apocalypse, Argentina.

Arte effymero. El apocalipsis como principio de creación en el cómic de la artista transgénero Effy Beth

En 2012, la artista de performance transgénero de origen israelí-argentino Effy Beth se preguntaba de forma retórica en la primera página de su único cómic, TRANSita rápido - TRANSita lento: "2012! Qué no es ahora el fin del mundo?" Según la interpretación New Age del calendario maya, el mundo terminaría el año 2012. Sin embargo, si la asociamos con las narrativas de no-ficción que exploran los retos que sufren las personas queer en nuestra sociedad, podemos entender la pregunta como algo más que una referencia circunstancial. En su cómic, Effy Beth narra la identidad transgénero como apocalíptica y, tal como ha hecho en sus obras anteriores, convierte la transgresión en un principio creador para poder desarrollar una poética y una política de lo efímero.

PALABRAS CLAVE: Effy Beth, cómic, narrativas queer, apocalipsis, Argentina.

Lorinquer-Hervé, Marie (2020), “Arte effymero. L'apocalypse comme principe de création dans la bande dessinée de l'artiste trans Effy Beth", Lectora, 26: 101-115. ISSN: 1136-5781 D.O.I.: 10.1344/Lectora2020.26.7, marie.lorinquer-herve@u-bordeaux-montaigne.fr

Recepció: 12 de desembre de 2019 - Acceptació: 31 de març de 2020 
Ils disent crise, nous disons révolution.

-Paul B. Preciado

TRANSita rápido - TRANSita lento (2012) est la seule bande dessinée d'Elizabeth Mía Chorubczyk (1988-2014) qui signait ses œuvres sous les pseudonymes d'Effy Beth ou Effymia. Formée à l'Instituto Universitario Nacional del Arte (IUNA) de Buenos Aires, l'artiste israélo-argentine s'est majoritairement consacrée aux performances, aux happenings et aux installations. Elle se définit comme une femme trans, "artiste conceptuelle, performeuse et féministe queer". 'Son identité de genre est au cœur de son travail, qui mêle l'intime et le politique sur le mode militant, et sa bande dessinée ne fait pas exception. Cette dernière s'inscrit dans une période de son œuvre qui fait une part plus grande au dessin. En 2007, Effy Beth effectue un séjour en hôpital psychiatrique en raison de désirs suicidaires et se met à dessiner depuis sa chambre (Máximo, 2016: 172). La bande dessinée qu'elle crée en 2012 se trouve donc dans la triple continuité de cette pratique du dessin, de la thématisation de son identité de genre et du mal-être auquel peut conduire cette identité dans le contexte sociopolitique contemporain. ${ }^{2}$

TRANSita rápido - TRANSita lento est un récit de non-fiction en deux volets qui fonctionnent en diptyque. Le premier volet, TRANSita rápido, est publié sur sa page Facebook tout au long du mois de janvier 2012. ${ }^{3}$ Il est constitué d'épisodes autoconclusifs qui explorent son quotidien de personne transgenre, fait de violences sociales et de difficultés socio-affectives. Le second volet, TRANSita lento, est publié en une seule fois, le 5 novembre de la même année. Il consiste en un récit en huit planches d'une période durant laquelle l'artiste avait affirmé son identité trans dans son cercle intime mais continuait à se présenter comme un homme cisgenre dans sa vie professionnelle par crainte de perdre son emploi.

Ce double récit s'ouvre sur une question rhétorique placée en page de couverture du premier volet: "2012! Qué no es ahora el fin del mundo?"

\footnotetext{
${ }^{1}$ Cette description apparait en bannière de l'un de ses blogs, Tengoeffymia.

${ }^{2}$ Si l'impact de cette loi sur l'expérience quotidienne des personnes transgenre est limitée, il faut souligner à cet égard que la première partie de la bande dessinée est parue avant le vote de la Ley de Identidad de Género, le 9 mai 2012. Celle-ci permet un changement d'état civil sans obligation de solliciter l'autorisation d'une autorité médicale ou judiciaire.

${ }^{3}$ La totalité de la bande dessinée a ensuite été publiée à titre posthume dans un recueil des travaux de l’artiste (Máximo, 2016). Les planches reproduites dans cet article sont tirées de cet ouvrage.
}

102

Lectora, 26 (2020): 101-115. ISSN: 1136-5781 D.O.I.: 10.1344/Lectora2020.26.7 


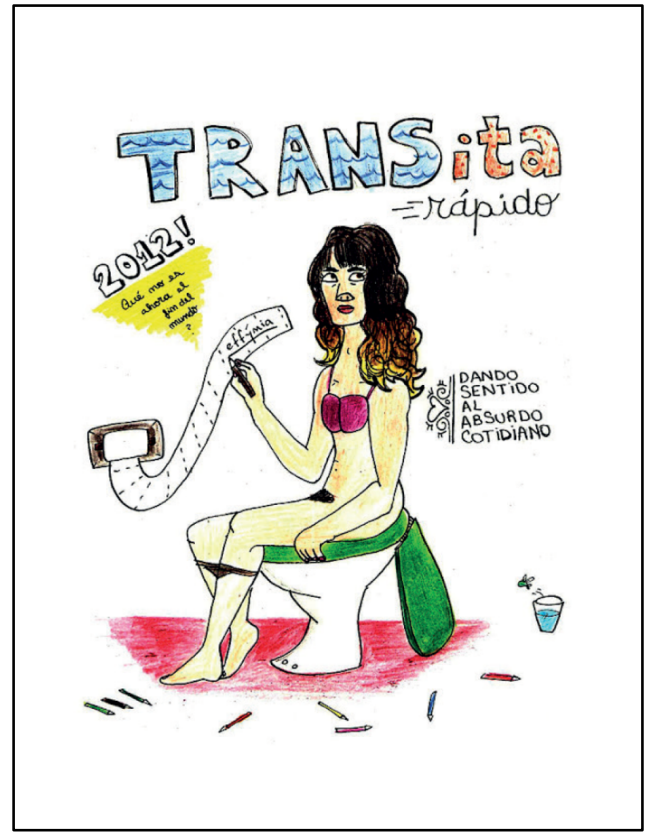

Illustration 1. Page de couverture.

La date de création de la bande dessinée coïncide en effet avec l'année qui, selon les interprétations New Age du calendrier maya, devait être celle de la fin du monde. Placée en introduction d'une œuvre qui insiste sur les difficultés quotidiennes imposées aux personnes queer, la question peut toutefois être lue comme davantage qu'un clin d'œil conjoncturel. Cet article sera une proposition de lecture de la bande dessinée au prisme de cette question liminaire. Il s'agira d'observer de quelle manière l'identité trans -et l'identité queer de manière générale- est narrée comme une identité apocalyptique. La référence à l'année 2012 mêlant dans la conscience collective les prédictions mayas et leurs interprétations catastrophistes, nous prendrons en compte les deux conceptions majeures et antagonistes de la notion d'apocalypse. La première, populaire, l'entend comme une catastrophe augurant de la fin du monde. La seconde, scripturaire, l'envisage au contraire comme une fin de cycle et, donc, comme une re-création. Nous verrons dans un premier temps que la construction du diptyque fait apparaitre le coming out comme une condamnation à une sortie du monde. Nous nous intéresserons ensuite à la manière dont la dynamique de répétition qui structure le récit du quotidien opère un renversement selon lequel la permanence du monde se mue en une fin des temps. Enfin, nous observerons de quelle façon l'artiste poursuit dans cette bande dessinée la 
construction d'une poétique (et d'une politique) de l'éphémère initiée dans ses performances. Afin de développer davantage cette réflexion sur les liens entre la notion d'apocalypse et les narrations queer, cet article sera également l'occasion d'une lecture croisée de la bande dessinée d'Effy Beth et du court métrage I Want to Kill Myself (2017) de l'artiste canadienne trans Vivek Shraya (1981-).

\section{Coming out - going out}

Au premier coup d'œil, il se dégage de la bande dessinée d'Effy Beth une évidente marginalité. L'artiste se tient à distance des codes de la bande dessinée classique et assume volontiers un certain amateurisme. Elle délaisse le "beau dessin" académique au profit d'un dessin à main levée parfois rudimentaire et qui témoigne d'une rapidité d'exécution. Le trait est vif et appuyé, les couleurs presque criardes, non mélangées, et les aplats toujours laissés à l'état d'une apparente esquisse. Il émane de son dessin ce que l'on pourrait appeler un bruit, par analogie avec le langage photographique. Le geste de la dessinatrice ne s'efface pas sous ce qu'il représente mais se manifeste avec vacarme en chaque endroit. Il met en exergue ce que Philippe Marion nomme la "graphiation", c'est-à-dire ce qui "avant de contribuer à la figuration, [...] est automonstration, dans la trace, d'une identité graphique perceptible dans la spécificité d'une empreinte" (1993: 36). À l'image de l'ensemble de son œuvre, la bande dessinée d'Effy Beth engage le corps, comme objet, comme outil et comme matériau. Ce choix d'énonciation graphique favorise chez le/la lecteur/ice le sentiment d'un dessin singularisé et littéralement étrange -ouvrant des perspectives de réflexion sur les potentialités queer du dessin. Visuellement, la "spécificité de l'empreinte" agit comme un premier coming out: elle fait apparaitre une étrangeté au regard d'un code de référence et inscrit donc d'emblée l'œuvre dans situation marginale. Cette singularité et cette expressivité du trait font songer au mouvement punk et à son mot d'ordre du do it yourself, qui s'est largement diffusé dans la bande dessinée underground anticonformiste des années 1960 et 1970 en Amérique du Nord —et notamment dans les récits autobiographiques (Justin Green, Robert Crumb, Art Spiegelman) et féministes et/ou LGBT (Mary Wings, les autrices des collectifs Wimmen's Comix et Twisted Sisters) - qui a ouvert la voie à une émancipation des codes de la bande dessinée dite "de genre" (expression que l’on pourrait entendre ici aussi bien dans son sens esthétique que dans un sens social) au cours des décennies suivantes en Amérique du Nord (Alison Bechdel, Julie Doucet) comme en Argentine, où les publications indépendantes se sont multipliées à partir de la seconde moitié des années 1980. Ces pas de côté vis-à-vis des canons en vigueur ne sont eux-mêmes pas sans évoquer les arts naïf et brut apparus quelques dizaines d'année plus tôt —deux courants qui sont les noms donnés par le secteur hégémonique de l'Art aux tableaux contrevenant à ses règles, soit que leurs auteur/trices aient une formation artistique non occidentale ou inexistante dans le cas de l'art naïf, soit qu'ils/elles soient

104

Lectora, 26 (2020): 101-115. ISSN: 1136-5781 D.O.I.: 10.1344/Lectora2020.26.7 
neuroatypiques dans le cas de l'art brut. Dans une bande dessinée à teneur autobiographique, la proximité du dessin d'Effy Beth avec ces productions a quelque chose d'un ancrage résolu dans la marge, en forme de retournement du stigmate. De fait, c'est précisément l'histoire de cette mise en marge qui fait l'objet du récit.

La première partie de la bande dessinée est constituée de trente-et-une chroniques publiées durant le mois de janvier 2012 sur la page Facebook d'Effy Beth, à raison d'une par jour. Sur le mode de l'anecdote ou de la réflexion théorique, ces pages rendent compte des violences opposées en société aux personnes transgenres, et aux personnes queer de manière générale. Sont ainsi racontées les difficultés rencontrées par l'autrice-narratrice dans ses relations affectives ou professionnelles, mais également des choses vues, telles qu'une conversation entre deux dames se demandant quel bracelet offrir au mari de l'une d'elles qui ne soit pas trop féminin et qui ne fasse pas peser sur lui le soupçon de l'homosexualité. Sont racontés le marketing genré qui pousse les enfants à s'organiser en groupes fermés, l'inadaptation du monde médical à certains corps et la façon dont un grand quotidien national titre un article "Travestis contentas con la mudanza a los bosques", ne faisant aucune différence entre expression de genre et prostitution. Au gré des trente-et-un épisodes sont ainsi égrenés les organismes, institutions et lois qui définissent une norme et un hors-norme en termes d'identité de genre. C'est-à-dire, les outils du maintien de ce que Monique Wittig (1992) nomme le régime politique hétérosexuel: une organisation sociale totalitaire fondée sur la naturalisation d'une différence entre les sexes. Le rythme quotidien de publication des planches donne à sentir la manière dont ces outils sont mobilisés chaque jour, dans chaque situation, pour rappeler à l'ordre les hors la loi. Ces tentatives de régulation tracent les frontières de l'ordre social et désignent par conséquent ceux qui les transgressent comme des dangers pour l'ordre du monde. En d'autres termes, la société est mise en scène comme percevant les identités queer comme des menaces apocalyptiques. C'est ce qu'illustre la chronique du 22 janvier 2012 intitulée "Crimen": l'autrice y recourt à l'imagerie nazie et se représente arborant l'étoile jaune et les triangles rose et marron, stigmates de la menace qu'elle représente.

Dans le second volet du diptyque, cette lecture du corps trans comme une menace est explorée de façon plus intime. Perçue comme un danger pour l'ordre du monde, et donc comme un indésirable à éloigner ou à réguler, l'identité queer se mue en menace pour qui la vit. Dans une longue tirade sur la première planche, le personnage d'Effy confie à sa mère sa résignation à se conformer aux injonctions sociales pour ne pas être marginalisée. Le texte est disposé dans une bulle qui occupe les deux tiers supérieurs de la planche et se poursuit en bas de la page, enserrant et écrasant symboliquement le visage du personnage. Cette figuration d'un étouffement de l'agentivité est répétée par le contraste chromatique. Dans une bande dessinée caractérisée par ses couleurs vives, l'autrice-narratrice s'autoreprésente en "hija gris que nadie ve", tel qu'elle le formule dans la bulle. La continuité ainsi établie entre les mots du texte et la 
représentation graphique donne à voir le modelage du corps par les normes sociales. Cette autoreprésentation incarne la manière dont le langage de "la matrice de pouvoir" est, selon Judith Butler, "producteur, constitutif, voire, pourrait-on soutenir, performatif, dans la mesure où cet acte signifiant délimite et trace les contours du corps dont il prétend ensuite qu'il précède toute signification" (Butler, 2009: 40-42). Le sujet qui ne se conforme pas à la lecture socialement admise de son corps se condamne à une disparition du monde intelligible. Les stratégies mises en place pour neutraliser la menace apocalyptique représentée par les identités trans conduisent à ce que l'on pourrait qualifier d'une apocalypse intime. Dans les planches suivantes, l'effacement du corps d'Effy se poursuit à travers la description des étapes du déguisement auquel elle se soumet tous les matins avant d'aller travailler. Puisque le traitement hormonal qu'elle suit entraine des transformations corporelles telles que le développement de la poitrine et des hanches, elle est contrainte, en plus de revêtir des tenues masculines, de se bander la poitrine et de porter des vêtements larges. Cette opération de camouflage qui a pour but de la faire passer pour un homme cisgenre finit donc par s'apparenter à une stratégie de passing adoptée par certains hommes transgenre.

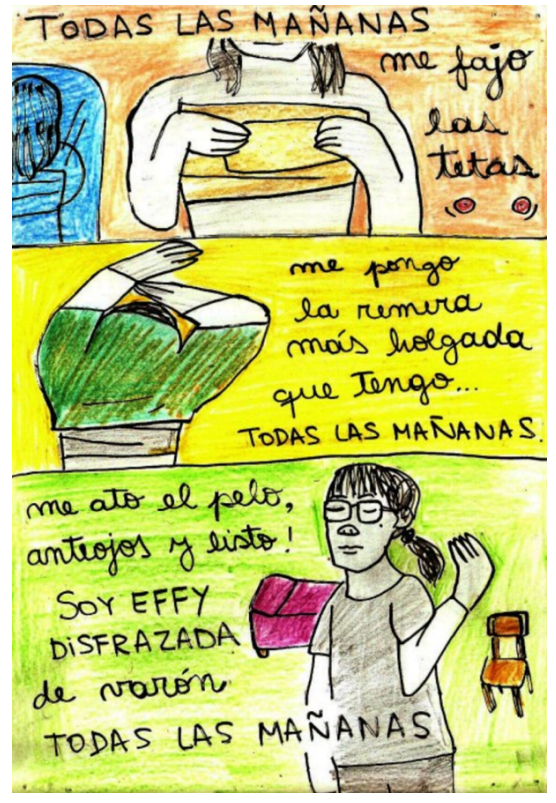

Illustration 2. Camouflage 1.

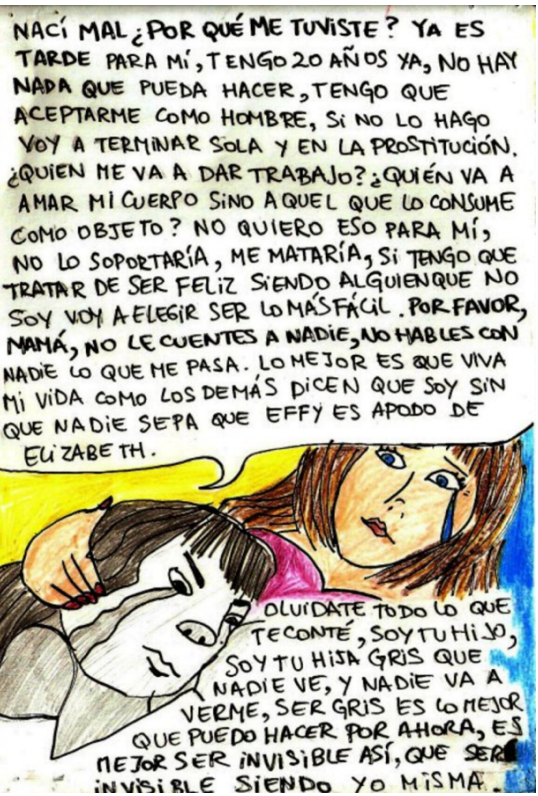

Illustration 3. Camouflage 2. 
Au lieu d'un supposé retour à un état de nature, la transformation est racontée comme une autre transition et le corps se trouve pris entre deux mouvements contraires, comme annulé.

Dans les dernières planches de la seconde partie, cette annihilation culmine dans le récit d'un désir suicidaire. L'autrice-narratrice raconte s'être couchée triste tous les soirs et avoir un jour demandé à Dieu avant de dormir: "Yo me voy a dormir ahora y vos te encargás de que no me vuelva a despertar". Cette prière revient presque mot pour mot dans le court-métrage de l'artiste canadienne trans Vivek Shraya, I Want to Kill Myself. Fait d'une suite de photographies et d'un texte prononcé en off, le court métrage s'ouvre sur des images du corps de l'artiste, accompagnées des phrases suivantes:

I wanted to kill myself when I was eleven. I learned I had a body through your condemnation of my body. Please god, don't let me wake up. I wanted to kill myself when I was thirteen. I often tried to suffocate myself with my pillow. Please god, don't let me wake up. (Shraya, 2017)

Dans chacune des deux œuvres, le processus de coming out transgenre est narré comme une condamnation à mort. De fait, les études réalisées sur le sujet évaluent à cinq à dix fois supérieur le risque de suicide chez une personne trans par rapport à une personne cis. ${ }^{4}$ Ces études soulignent que cette détresse n'a, bien entendu, rien d'une disposition particulière aux troubles psychiques mais résulte directement de l'expérience de la violence des mécanismes de désinsertion sociale. C’est ce que Vivek Shraya énonce explicitement à travers la phrase "I learned I had a body through your condemnation of my body". Et c'est ce qui se dégage de la manière dont Effy Beth construit son diptyque. Au moment de la mettre en ligne, l'autrice a présenté la seconde partie de la bande dessinée comme une prequel de la première. L'épisode qui y est raconté est antérieur aux expériences de rejet décrites dans le premier volet. Dans l'ordre de la lecture, en revanche, la confrontation aux mécanismes d'exclusion vient avant le désir suicidaire que déclenche la période du coming out. Ce désir apparait donc comme une conséquence des violences de la première partie. Cette inversion rappelle l'idée énoncée par Didier Éribon en ouverture de Réflexions sur la question gay selon laquelle "au commencement, il y a l'injure" (Éribon, 1999: 29). Autrement dit, l'injure est présente et déterminante immédiatement, avant même sa profération. La rencontre avec l'insulte "ne fait que symboliser, condenser, les multiples rencontres

\footnotetext{
${ }^{4}$ Jody L. Herman, Ann P. Haas, Philip L. Rodgers (2014). L'étude a été menée aux États-Unis et est à ce jour la seule base de données d'une telle envergure sur la question. Sur la situation particulière des personnes trans en Argentine, voir l'enquête menée par la fondation Huésped et la Asociación de Travestis, Transexuales y Trangéneros de Argentina (ATTTA) en 2013 suite à l'adoption de la Ley de Identidad de Género en mai 2012: Fundación Huésped, ATTTA (2014).
} 
avec le regard insultant, les mots blessants, les images offensantes, que la vie quotidienne réserve aux individus appartenant à des catégories stigmatisées" (Éribon, 2015: 83). Ces "verdicts" du discours hégémonique, qui posent les limites du monde et configurent certaines identités comme dissidentes, fonctionnent comme des prophéties apocalyptiques. La manifestation de l'une de ces identités condamnera inéluctablement qui l'incarnera car elle le poussera hors des limites du monde.

\section{"El resto es máquina. Y yo no"}

La disposition anachronique des deux parties fait par ailleurs de la bande dessinée une œuvre circulaire. Le fait que la première partie soit chronologiquement la suite de la seconde favorise chez le/la lecteur/trice un sentiment de perpétuel recommencement. Ce sentiment est d'autant plus fort que le format bref des chroniques de la première partie et leur publication quotidienne sur Facebook impriment une cadence régulière à la lecture. Les commentaires laissés par les lecteur/trices sur les différentes planches

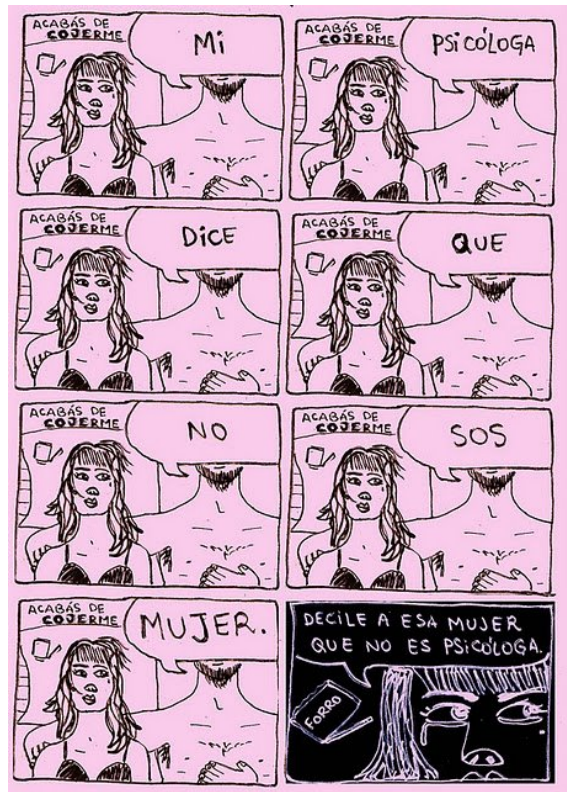

Illustration 4. "Una suerte de Andy Warhol's Car Crash Experience". au moment de leur publication permettent en effet de vérifier cette ritualisation de la lecture, souvent décrite comme accompagnant le petit-déjeuner. La mise en place de cette routine travaille à souligner le caractère répété de l'expérience narrée. La récurrence de la violence est mise en scène comme faisant partie de l'expérience de la violence. C'est ce que met en exergue une planche de la première partie intitulée "Una suerte de Andy Warhol's Car Crash Experience".

L'autrice-narratrice s'y représente couchée dans un lit aux côtés d'un homme avec lequel elle vient d'avoir un rapport sexuel. La planche à fond rose uni est organisée en un gaufrier de huit cases dont les sept premières sont quasiment identiques. Seul varie le contenu de la bulle dans laquelle apparaissent successivement chacun des sept mots de la phrase prononcée par l'homme: "Mi

\footnotetext{
5 “[C]es sentences dont on ne sait pas pourquoi elles ont été rendues, mais dont on découvre un jour qu'elles nous ont précédés et qu'elles nous enveloppent, nous accompagnent, nous jugent, nous condamnent sans qu'il y ait d'explication. La société comme verdict" (Éribon, 2013: 152).
}

108

Lectora, 26 (2020): 101-115. ISSN: 1136-5781 D.O.I.: 10.1344/Lectora2020.26.7 
psicóloga dice que no sos mujer". Cette dilatation dramatise le moment de la profération de la sentence violente en même temps qu'elle indique sa banalité et favorise une perte de la notion du temps chez le/la lecteur/trice, qui ne peut déterminer s'il s'agit là d'un seul moment que la narration étend, ou s'il s'agit d'une énumération de moments vécus à l'identique. Le procédé est emprunté à la série de tableaux Death and Disasters d'Andy Warhol, et plus particulièrement aux car crashes avec lesquels le titre de la planche indique une intericonicité. Dans ces tableaux, l'artiste étatsunien sérigraphie plusieurs fois la même image en noir et blanc d'un accident de voiture. À travers l'accumulation qui rappelle les pages des tabloïds, il met en tension deux temporalités: celle, cyclique, de la violence vécue et celle, linéaire, de l'indifférence qu'elle suscite. La réécriture d'Effy Beth invite à penser la négation répétée de son identité de genre comme autant de "morts et désastres" quotidiens, dont l'effrayante banalité est soulignée par la disparition du visage de l'homme, ou des hommes, derrière la bulle portant le discours dominant dont il n'est, ou dont ils ne sont, en dernière instance que le porte-voix. Tandis que les paroles prononcées mettent en doute l'identité d'Effy Beth, c'est bien l'identité de celui qui les prononce qui s'efface.

Cette anonymisation autorise une seconde lecture de la question liminaire “ ¿Qué no es ahora el fin del mundo?" La permanence jalousement protégée du monde est dépeinte comme un immobilisme et, donc, comme une fin des temps. L'apocalypse que la société redoute, au sens de fin du monde, est déjà là, "ahora". La planche intitulée "Stop clicking my balls" fait de l'ordre social une machine enrayée qui reproduit ad nauseam le même discours.

Au dessus des boutons d'un radiocassette dont la touche lecture est restée enfoncée, six personnages apparaissent dans six cases différentes. Ils enjoignent tour à tour une ou des personnes de "hacer el click", c'est-à-dire d'adhérer au discours hégémonique et d'abandonner leurs convictions hérétiques. Une mère essaie ainsi de convaincre une femme qui ne veut pas d'enfants qu'elle finira par réaliser que la maternité est son destin naturel, tandis qu'une lesbienne affirme que sa compagne bisexuelle se rendra compte tôt ou tard qu'elle est en réalité homosexuelle. À l'exception du

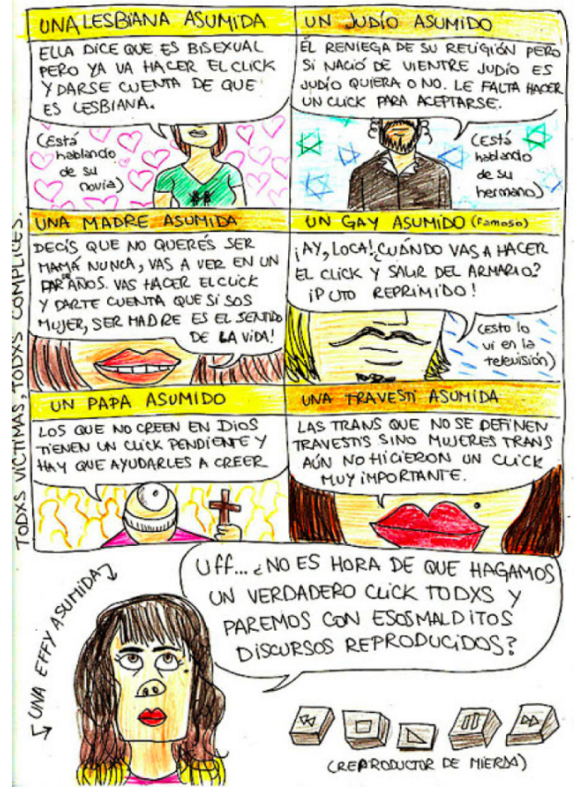

Illustration 5. "Stop clicking my balls". 
pape, qui apparait dans la cinquième case, les personnages mobilisés sont tous issus de minorités sexuelles, religieuses et/ou de genre. Le recours à ces figures sert d'une part de rappel que personne n'est à l'abri de reproduire le discours de la norme, tel que l'indique la légende "todxs víctimas, todxs cómplices" inscrite sur le bord gauche de la planche. D'autre part, le fait de placer ce discours dans la bouche de certaines de ses victimes les plus évidentes amplifie l'effet de répétition. Il donne à voir une sclérose qui s'étend à toutes les couches de la société. La dynamique de répétition travaillée par Effy Beth opère en somme un renversement: l'ordre du monde apparait menacé par sa propre permanence. L'absence d'une apocalypse, au sens scripturaire de fin de cycle et de renouvellement, conduit à une apocalypse entendue au sens courant de fin du monde.

De la même façon, dans I Want to Kill Myself, Vivek Shraya fait le récit de sa vie de personne queer en répétant comme une litanie "I wanted to kill myself". À la toute fin du court-métrage, elle change la formule en "Saying I want to kill myself kept me alive". La phrase fait d'abord référence aux vertus thérapeutiques de la confession de son désir suicidaire à ses proches. Cependant, elle peut également être entendue comme faisant référence au court-métrage lui-même. Le fait de répéter "I wanted to kill myself" renverse la menace et agit comme un révélateur. C'est la permanence du monde, qui produit inlassablement les mêmes effets, qui apparait comme mortifère.

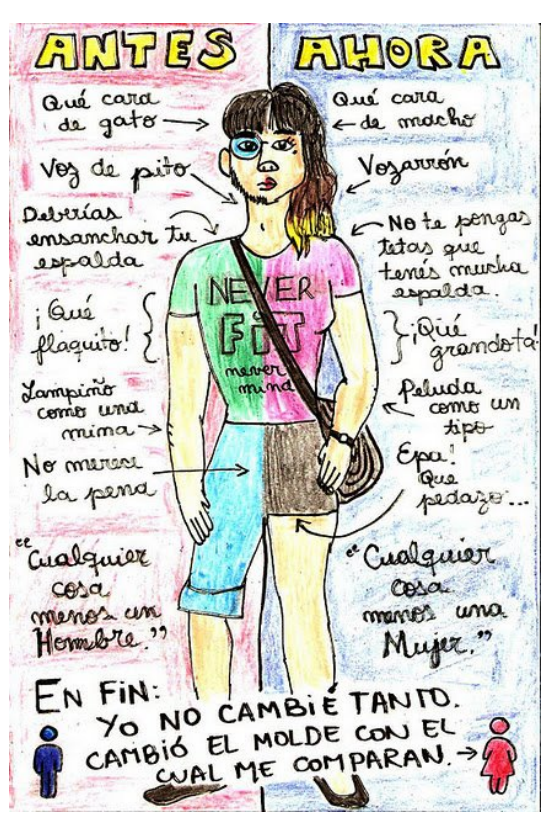

Illustration 6. "Sobre moldes". Par conséquent, l'émancipation de ce monde est simultanément légitimée et enclenchée par la répétition de la formule. Chez Effy Beth, cette transmutation de la fin $\mathrm{du}$ monde en horizon désirable est illustrée à travers la preuve par l'absurde. Dans la planche intitulée "Sobre moldes", l'autrice-narratrice s'autoreprésente en pied, sur toute la hauteur de la page et scindée en deux par un axe vertical, faisant songer aux planches anatomiques scientifiques.

Elle se figure sous une apparence d'homme sur la partie gauche légendée "Antes", et sous une apparence de femme sur la partie droite légendée “Ahora”. D’un côté et de l'autre de la page sont inscrites les réflexions suscitées par chacune des parties de son corps avant et après sa transition de genre. Ces réflexions sont à la fois semblables sur la forme et antagonistes sur le

110

Lectora, 26 (2020): 101-115. ISSN: 1136-5781 D.O.I.: 10.1344/Lectora2020.26.7 
fond. À la stigmatisation d'une "voz de pito" lorsque le corps scruté est perçu comme masculin répond ainsi celle d'un "vozarrón” lorsqu'il est perçu comme féminin. La multiplication de ces verdicts contraires appliqués à un même corps rend caduques les catégories (les moules) en fonction desquelles il est jugé. A rebours de l'imagerie scientifique mobilisée, cette multiplication met en scène un ordre du monde inopérant, révélé par l'identité trans. Dans la bande dessinée comme dans le court-métrage, la dissidence d'avec l'ordre du monde est donc présentée comme une apocalypse salvatrice - rappelant les derniers vers du poème "Soy" de la poétesse trans argentine Susy Shock, paru trois ans plus tôt: "El resto es máquina. Y yo no".

\section{L'apocalypse comme geste créateur}

À l'image du reste de l'œuvre d'Effy Beth, TRANSita rápido - TRANSita lento est en somme pensée sur le mode de l'intervention, au sens artistico-politique du terme. De la même manière que ses performances et happenings sont des productions éphémères, documentées a posteriori sur différents blogs mais inaccessibles dans leur forme première pour qui n'en a pas été le/la spectateur/trice in vivo, sa bande dessinée est située. Le choix de la publier à un rythme quotidien et sur un réseau social l'ancre dans un moment précis. Il s'agit d'intervenir dans ce moment, c'est-à-dire de le documenter et de le transformer à la fois. Le geste artistique est davantage investi comme un acte que comme la production d'un objet pérenne. En ce sens, la pratique d'Effy Beth a quelque chose d'apocalyptique. Elle se conçoit comme un basculement d'un moment à un autre. Dans un happening réalisé en 2011, intitulé El arte alimenta, l'artiste faisait de ce geste apocalyptique une éthique de la création artistique. Elle avait parcouru Buenos Aires pour proposer aux directeurs de musées de lui acheter pour 25 centimes de pesos des autocollants qui consistaient en la photographie de l'un de ses tétons, qu'elle se chargerait d'installer elle-même. Ces autocollants étaient une façon d'inviter à la réflexion quant au caractère "nourricier" de l'art, dans un sens symbolique pour le/la spectateur/trice et économique pour l'artiste. Ils étaient également une opération de transfiguration de sa poitrine de femme transgenre qui ne nourrirait jamais d'enfant mais qui acquérait dans l'œuvre une autre forme de fertilité. Face au refus de l'écrasante majorité des musées, qui lui opposaient la nécessité de soumettre d'abord un dossier à une commission, Effy Beth avait prolongé l'œuvre en rédigeant notamment une lettre à l'intention des quelques directeurs qui avaient accepté sa proposition. Elle expliquait l'annulation du projet et concluait la lettre par: "Le pido disculpas y le agradezco por ser una de las pocas personas abiertas al arte en el circuito del arte". Autrement dit, elle attirait l'attention sur une sclérose des institutions artistiques qui favorisaient la reproduction de formes consacrées au détriment d'initiatives déviantes. 
On comprend dès lors l'intérêt qu'a pu représenter l'investissement d'une bande dessinée autogérée. Lorsqu'elle publie TRANSita rápido - TRANSita lento sur Facebook, Effy Beth entre comme par effraction dans la bande dessinée. Cette incursion dans une forme artistique qu'elle n'avait jamais exploitée jusqu'alors est pour partie favorisée par l'état du champ de la bande dessinée argentine en 2012. Le marché éditorial local est alors dans une situation que l'on pourrait qualifier de postapocalyptique, pour filer la comparaison. Il s'est écroulé dans les années 90, sous l'effet conjugué de ses fragilités internes et des politiques économiques ménémistes, entrainant la disparition de la quasi-totalité des maisons d'édition. Pour continuer à exercer, les auteur/trices ont été contraint/e/s ou bien de travailler pour l'étranger, ou bien de s'en remettre à l'autogestion. Des formats alternatifs à la revue et à l'album ont donc connu un essor considérable: le fanzine d'abord, puis le blog. Par défaut, ces formats ont émancipé (pour partie au moins) la bande dessinée de sa dépendance aux circuits traditionnels de l'édition. En supprimant la nécessité de plaire à un éditeur d'abord, et d'avoir le bagage nécessaire pour respecter son cahier des charges ensuite, ils ont favorisé l'incursion dans la bande dessinée d'artistes et d'amateur/trices jusque là extérieurs au champ. Ces approches de la bande dessinée se sont ensuite largement développées dans les années 2000 sous les gouvernements des Kirchner et sous l'effet de l'avènement de l'internet 2.0. Lorsqu'Effy Beth se prête au jeu, elle renouvelle sa propre pratique en même temps qu'elle se joint ponctuellement à ce mouvement qui tend à défaire le genre de la bande dessinée telle qu'elle avait existé jusqu'alors. Elle participe à encourager une relecture de la crise du secteur comme une fin de cycle davantage que comme un chant du cygne.

Effy Beth investit la forme déviante comme une stratégie de mise en récit et d'affirmation d'une identité queer. Le récit de soi passe par la manifestation de cette étrangeté. Il se construit dans une logique de distinction d'avec un ordre établi. Plus encore, cette distinction s'étend au sujet lui-même et engendre une plasticité du récit de soi. Dans TRANSita rápido - TRANSita lento, Effy Beth revient sur des événements de sa vie déjà abordés dans de précédentes œuvres, voire recourt directement à l'autocitation. Ainsi, sur une planche de la seconde partie, les vêtements masculins que l'autrice-narratrice porte au travail sont disposés sur une chaise. Au dessus, le texte indique: "MI DISFRAZ me espera todas las mañanas sobre la silla". Visuellement et verbalement, la planche est une citation de l'installation "Mi disfraz" (octobre 2010) réalisée à l'occasion d'un festival. L'artiste avait exposé sur une chaise les vêtements qu'elle porterait le lendemain au travail, accompagnés d'un écriteau indiquant en lettres capitales: "MI DISFRAZ". De même, la planche sur laquelle l'autrice-narratrice arbore l'étoile jaune et les triangles rose et marron nazis est une autocitation du happening "La identidad como estigma" (octobre 2011) et les pages dédiées à la relation qu'elle a entretenue avec son supérieur hiérarchique ou son précepteur du judaïsme reprennent des récits proposés dans la performance "Unos 
cuantos piquetitos a la sirena" (septembre 2011) - pour ne mentionner que quelques exemples. Bon nombre des histoires racontées dans la bande dessinée sont des réécritures de précédents récits de soi. Le fait même de se prêter à ces réécritures n'est par ailleurs pas sans faire songer au cycle de performances "Mira colectiva" (aussi appelé "Soy tu re-creación") durant lequel Effy Beth posait et demandait aux spectateurs/trices de la dessiner, récoltant finalement pas moins de 800 versions d'elle-même. Cette re-création est un procédé que Vivek Shraya travaille également dans son œuvre: les périodes abordées dans I Want to Kill Myself recoupent des récits proposés dans ses œuvres précédentes (et suivantes), sous des formes aussi variées que le recueil de poèmes, de textes brefs, la série de photographies ou l'essai. Chez l'une et l'autre des deux artistes, le geste créatif mis en exergue est donc également un geste de création de soi. Le récit de l'affirmation d'une identité queer se fait à travers le renouvellement permanent. Il rejoint la définition de la transidentité proposée par le philosophe transgenre Paul B. Preciado:

Être trans, c'est désirer un processus de "créolisation" interne: accepter qu'on n'arrive à être soi que grâce au changement, à la mutation, au métissage. La voix que la testostérone propulse dans ma gorge n'est pas celle d'un homme, c'est la voix de la traversée. La voix qui tremble en moi est la voix de la frontière. "Nous comprenons mieux le monde, dit Glissant, lorsque nous tremblons avec lui, car le monde tremble dans toutes les directions". (Preciado, 2019: 34)

Si la "créolisation" — concept emprunté au philosophe Édouard Glissant - est au cœur de ces récits de soi, c’est qu'elle est envisagée non seulement comme propre de l'expérience transgenre, mais comme condition sine qua non, pour tout être, de l'accession à une subjectivation. L'identité se dessine au moyen d'une re-création permanente. Se tisse ainsi dans TRANSita rápido - TRANSita lento une poétique (et une politique) de l'éphémère. La possibilité de fréquentes apocalypses au sens scripturaire de renouvellements est un principe de création de soi autant qu'un principe d'écriture. Ce sont ces tremblements qui préservent d'une apocalypse entendue au sens courant de limite et de condamnation. C'est ce qu'Effy Beth rappellera en des termes très proches dans une courte note laissée sur sa page Facebook avant son suicide en 2014: "Que el mundo tiemble, cuando yo no tiemble".

\section{Conclusion}

En guise de conclusion, on peut se souvenir du t-shirt qu'arborait Effy Beth en 2010 lors de la marche des fiertés de Buenos Aires. On pouvait y lire "Transexual, bisexual, casta, judía, atea, extranjera, porteña, artista, mujer. Potencial amenaza a tus prejuicios". À l'image de cette affirmation, le récit de soi élaboré dans TRANSita 
rápido - TRANSita lento est un récit de résistance et de déconstruction d'un ordre du monde. C'est ce qu'indiquent les différentes interprétations possibles de la question rhétorique placée en page de couverture. Elle peut faire allusion à la désignation de l'identité queer comme la menace d'une catastrophe annonciatrice de fin du monde. Elle peut également être envisagée comme une façon de se demander si cette fin du monde redoutée n'est pas déjà vécue "ahora", si elle n'est pas consubstantielle de l'ordre du monde jalousement conservé. Elle peut encore être comprise comme l'expression d'une impatience et l'annonce d'une impulsion donnée à un renouvellement. Enfin, elle peut être lue comme tout cela à la fois. C'est en effet dans l'articulation de ces trois lectures que se tisse ce que Pablo Oscar Farneda nomme une "pratique de soi" queer. Dans sa thèse dédiée aux expressions artistiques trans actuelles à Buenos Aires, le critique définit cette pratique comme un ensemble de modalités autoreprésentatives "que no se ajustan totalmente a una categoría auto-biográfica, sino más bien a la puesta en variación del propio cuerpo y las experiencias vitales en función de un extrañamiento, más aún que de reconocimiento" (Farneda, 2014: 7). Le récit de soi devient alors le lieu d'une élaboration de soi qui prend chez Effy Beth la forme apocalyptique d'une recréation perpétuelle.

\section{RÉFÉRENCES BIBLIOGRAPHIQUES}

Beth, Effy (2012), “TRANSita rápido - TRANSita lento”, Facebook, 31/10/2019. $<$ https://www.facebook.com/tengoeffymia/media_set?set=a.139489686165957\& type $=3>$

-(s.d.), Tengoeffymia, 31/10/2019. <http://tengoeffymia.blogspot.com/>

Butler, Judith (2009), Ces corps qui comptent. De la matérialité et des limites discursives $d u$ «sexe», Paris, Amsterdam.

Éribon, Didier (1999), Réflexions sur la question gay, Paris, Fayard.

-(2013), La société comme verdict, Paris, Fayard.

-(2015), Une morale du minoritaire. Variations sur un thème de Jean Genet, Paris, Flammarion.

Farneda, Pablo Oscar (2014), Prácticas de Sí. Subjetividades contemporáneas en las expresiones artísticas trans actuales en Buenos Aires, Buenos Aires, Filodigital.

Fundación Huésped, ATTTA, ONUSIDA (2014), Ley de identidad de género y acceso al cuidado de la salud de las personas trans en Argentina. Resumen ejecutivo. <https://www.huesped.org.ar/wp-content/uploads/2015/01/Ley-deidentidad-de-genero-y-acceso-al-cuidado-de-la-salud-personas-trans-enArgentina.pdf $>$

Herman, Jody L., Ann P. Haas and Philip L. Rodgers (2014), Suicide Attempts among Transgender and Gender Non-Conforming Adults, New York \& Los Angeles, 
American Foundation for Suicide Prevention \& the UCLA William Institute. <https://escholarship.org/uc/item/8xg8061f >

Marion, Philippe (1993), Traces en cases. Travail graphique, figuration narrative et participation du lecteur (essai sur la bande dessinée), Louvain-la-Neuve, Academia.

Máximo, Matías (2016), Que el mundo tiemble. Cuerpo y performance en la obra de Effy Beth, La Plata, EDULP.

Preciado, Paul B. (2019), Un appartement sur Uranus, Paris, Grasset.

Shock, Susy (2011), Poemario Trans Pirado, Buenos Aires, Nuevos Tiempos.

Shraya, Vivek (2017), I Want to Kill Myself, 31/10/2019. <https://vivekshraya.com/ projects/visual/i-want-to-kill-myself/>

Wittig, Monique (2018), La pensée straight, Paris, Amsterdam. [1992] 